\title{
Meikin is a conserved regulator of meiosis I-specific kinetochore function
}

Jihye $\mathrm{Kim}^{1}{ }^{*}$, Kei-ichiro Ishiguro ${ }^{1 *}$, Aya Nambu ${ }^{1}$, Bungo Akiyoshi ${ }^{1}$, Shihori Yokobayashi ${ }^{1}$, Ayano Kagami ${ }^{1}$, Tadashi Ishiguro ${ }^{1}$, Alberto M. Pendas ${ }^{2}$, Naoki Takeda ${ }^{3}$, Yogo Sakakibara ${ }^{4}$, Tomoya S. Kitajima ${ }^{4}$, Yuji Tanno ${ }^{1}$, Takeshi Sakuno ${ }^{1}$, Yoshinori Watanabe ${ }^{1}+$

${ }^{1}$ Laboratory of Chromosome Dynamics, Institute of Molecular and Cellular Biosciences, University of Tokyo, 1-1-1Yayoi, Tokyo 113-0032, Japan

${ }^{2}$ Instituto de Biología Molecular y Celular del Cáncer (CSIC-USAL), 37007 Salamanca, Spain

${ }^{3}$ Center for Animal Resources and Development, Kumamoto University, 2-2-1 Honjo, Kumamoto 860-0811 Japan

${ }^{4}$ Laboratory for Chromosome Segregation, RIKEN Center for Developmental Biology, 2-2-3

Minatojima-Minamimachi, Chuo-ku, Kobe 650-0047, Japan

† To whom correspondence should be addressed. E-mail: ywatanab@iam.u-tokyo.ac.jp

Telephone: +81-3-5841-1467

FAX: $+81-3-5841-1468$

*These authors contributed equally to this work.

The kinetochore is the crucial apparatus regulating chromosome segregation in mitosis and meiosis. Particularly in meiosis I, unlike in mitosis, sister kinetochores are captured by microtubules emanating from the same spindle pole (mono-orientation) and centromeric cohesion mediated by cohesin is protected in the following anaphase. Although meiotic kinetochore factors have been identified only in budding and fission yeasts, these molecules and their functions are thought to have diverged earlier. Therefore, a conserved mechanism for meiotic kinetochore regulation remains elusive. Here we have identified meiosis-specific kinetochore factor MEIKIN in mouse, which functions in meiosis I but neither in meiosis II nor in mitosis. MEIKIN plays a crucial role in both mono-orientation and centromeric cohesion protection, partly by stabilizing the localization of the cohesin protector shugoshin. These functions are mediated largely by the activity of Polo-like kinase PLK1, which is enriched to kinetochores depending on MEIKIN. Our integrative analysis indicates that MEIKIN is the long awaited key regulator of meiotic kinetochore function, which is conserved from yeasts to humans. 
In mitosis, sister chromatid cohesion is established depending on cohesin in S phase and maintained until metaphase when the sister chromatids are captured by spindle microtubules from opposite poles and aligned on the spindle equator. For the onset of anaphase, the anaphase-promoting complex (APC) triggers the degradation of securin, an inhibitory chaperone for separase that cleaves cohesin RAD21 and removes cohesin along the entire chromosome. This removal of cohesin triggers the separation of sister chromatids and their movement to opposite poles, a process called equational division ${ }^{1-3}$. During meiosis, however, meiotic cohesin REC8 largely replaces RAD21 along the entire chromosomes; one round of DNA replication is followed by two rounds of nuclear division, which results in four haploid nuclei or gametes (Fig. 1a).

In the first division of meiosis (meiosis I), homologous chromosomes connected by chiasmata are captured from the opposite poles, while sisters are captured from the same pole (mono-orientation). At the onset of anaphase I, REC8 cohesin is cleaved by separase along the arm regions, but protected at centromeres until metaphase $\mathrm{II}^{4-6}$. Thus, mono-orientation and centromeric cohesion protection are two hallmarks of meiotic kinetochore function, which are widely conserved among eukaryotic organisms ${ }^{7-9}$ (Fig. 1a). There is accumulating evidence that cohesion protection is mediated by the centromeric protein shugoshin (SGO) and its partner protein phosphatase $2 \mathrm{~A}(\mathrm{PP} 2 \mathrm{~A})^{10-15}$, which antagonizes REC8 phosphorylation, a prerequisite of cleavage ${ }^{16,17}$. So far, meiosis-specific kinetochore proteins have been identified only in two yeasts (S. cerevisiae Spo13 and Mam1 (monopolin subunit), and $S$. pombe Moa1) ${ }^{18-23}$. Puzzlingly, however, because their structural and functional similarities remain to be identified, conservation of meiotic kinetochore regulation is questionable even between yeasts $^{8,9}$. Therefore, in this study, we address the long-standing question of whether meiotic kinetochore regulation is conserved from yeasts to mammals, and, if so, how.

\section{Mammalian meiotic kinetochore protein MEIKIN}

Fission yeast Moa1 interacts directly with the conserved kinetochore protein Cnp3 (CENP-C homolog), and localizes to the kinetochore in meiosis $\mathrm{I}^{24}$. To identify an equivalent meiosis-specific kinetochore protein in mammals, we searched for proteins that interact with mouse CENP-C in yeast two-hybrid assay using a cDNA library prepared from mouse testis (Extended Data Fig. 1a). The most frequently obtained clones (4930404A10Rik gene) encoded a novel protein, which we named MEIKIN (Meiosis-specific kinetochore protein) after its molecular nature (see below). Meikin shows specific expression in germ cells (both testis and ovary) but not in other organs (Extended Data Fig. 1b). Immunoprecipitation assays using testis chromatin extracts indicate that MEIKIN indeed forms a complex with CENP-C 
(Extended Data Fig. 1c). Blast search analysis revealed that MEIKIN is a new protein conserved among vertebrates (Extended Data Fig. 2).

To determine the localization of MEIKIN, we immunostained for MEIKIN in spermatocytes along with SYCP3, a component of axial element, and ACA (anti-centromeric antibodies), which stains constitutive centromeric proteins including CENP-C (Fig. 1b, Extended Data Fig. 3a). MEIKIN appears at centromeres during pachytene stage when homologous chromosomes (homologs) are synapsed. Centromeric MEIKIN signals culminate during diplotene stage, persist with gradual reduction until metaphase I, and finally disappear in anaphase I. In meiosis II, MEIKIN does not reappear on chromatins. This localization contrasts to that of ACA (or CENP-C), which increases during zygotene and persists throughout meiosis I and meiosis II (Fig. 1b and Extended Data Fig. 3a). A similar localization pattern of MEIKIN was observed in oocytes (Extended Data Fig. 3b, c). To delineate the mechanism of MEIKIN localization, we narrowed down the kinetochore localization sequences of MEIKIN by expressing their GFP-fusion versions in testis, and identified that the C-terminus conserved sequences play an essential role in localizing to kinetochores (Fig. 1c, Extended Data Fig. 1d). We conclude that MEIKIN is a meiosis I-specific kinetochore protein whose localization is mediated by its interaction with CENP-C.

\section{Meikin KO mice are infertile}

To address the function of MEIKIN in meiosis, we generated $\mathrm{Meikin}^{-/-}$mice (Extended Data Fig. 4a-c). Immunostaining of spermatocytes confirmed that MEIKIN proteins are absent from the kinetochores in Meikin $^{-/-}$(Fig. 1d). Although homozygous Meikin ${ }^{-/-}$mice develop normally and exhibited no overt phenotype, both male and female KO (knockout) mice are completely infertile (data not shown). Cytological inspection of testis and ovary detected no obvious difference between wild-type and $\mathrm{Meikin}^{-/-}$mice, although mature sperms are rarely observed in the epididymis of Meikin ${ }^{-/-}$mice (Extended Data Fig. 4d-g). Immunostaining of spermatocytes revealed apparently normal meiotic prophase progression as the structures of the axial elements (AEs) are properly formed and develop into synapsis in pachytene stage and further into condensed meiotic chromosomes (Fig. 1d, see below). The round spermatids produced after meiosis, however, become enlarged, because meiosis II was somehow skipped (Fig. 1e). These results suggest that infertility in male $\mathrm{Meikin}^{-/-}$mice originates from defects in meiotic chromosome segregation and subsequent spermatogenesis rather from a meiotic prophase aberration (see below).

\section{MEIKIN protects centromeric cohesion}


Female Meikin $^{-/-}$mice produced germinal vesicle (GV) stage oocytes similar to wild-type mice presumably as a result of normal prophase progression. We then examined meiotic division by isolating GV-stage oocytes. When placed in culture medium, the GV-stage oocytes resume meiosis, undergo germinal vesicle breakdown (GVBD), form meiosis I spindles and align bivalent chromosomes using their chiasmata. When all bivalents are aligned and come under tension, chiasmata are resolved and homologs are segregated to opposite poles. Because one set of chromosomes is retained in the oocytes but the other is segregated into the first polar body (PB), meiosis I division can be monitored by the extrusion of the first PB. Light microscopy observation revealed that Meikin $^{-/}$oocytes extrude first PB on average $2 \mathrm{~h}$ later than oocytes from wild-type littermates, although most mutant oocytes ( $>$ 90\%) eventually enter meiosis I division (Fig. 2a). In wild-type oocytes, bivalents are aligned by $6 \mathrm{~h}$ after GVBD at the spindle equator, thus forming a metaphase plate (Fig. 2b). Although Meikin $^{-/}$oocytes also show bivalent alignment in metaphase I, chromosome congression is partly perturbed (Fig. 2b). Because inactivation of the spindle assembly checkpoint (SAC) cancels the delay in anaphase onset of $\mathrm{Meikin}^{-/-}$oocytes compared to wild-type oocytes (Extended Data Fig. 5), we reasoned that chromosome alignment defects and consequent activation of the SAC might be the reason for the delay in the onset of anaphase I.

To analyze the spatiotemporal dynamics of kinetochores and chromosomes during meiosis I, we recorded four-dimensional (4D) datasets of kinetochores and chromosomes labeled with 2mEGFP-CENP-C and Histone 2B (H2B)-mCherry ${ }^{25}$. We followed the frames at the onset of anaphase in wild-type and $\mathrm{Meikin}^{-/-}$oocytes and confirmed that the bivalents aligned in the metaphase plate are indeed separated in anaphase I in both oocytes (Fig. 2c). Strikingly, however, many pairs of sister kinetochores start to split at the onset of anaphase in Meikin ${ }^{-/-}$ oocytes but rarely in wild-type oocytes (Fig. 2c). Accordingly, chromosome alignment in metaphase II is largely disrupted in $\mathrm{Meikin}^{-/-}$oocytes (Fig. 2d), presumably because sister chromatids are prematurely separated. Indeed, immunostaining of spread chromosomes revealed that centromeric REC8 signals are lost from metaphase II chromosomes in Meikin ${ }^{-/-}$ oocytes, whereas REC8 localizes normally to the interchromatid axes on metaphase I bivalents (Fig. 2e). The loss of centromeric cohesin REC8 (and cohesion) during anaphase I is reminiscent of that in mice defective in shugoshin-2 (SGO2), the protector of centromeric cohesin in mouse meiosis $^{11,12}$ (Fig. 2e). We then examined bivalents for SGO2 localization, which usually appears at centromeres in metaphase $\mathrm{I}^{11}{ }^{12}$. Intriguingly, SGO2 localization is diminished in Meikin ${ }^{-/-}$oocytes (Fig. 2f), possibly explaining the impaired protection of centromeric cohesion in anaphase I. Similar defects were observed in $\mathrm{Meikin}^{-/-}$ spermatocytes (Extended Data Fig. 6a). Notably, kinetochore separation during anaphase I is 
milder in $\mathrm{Meikin}^{-/-}$spermatocytes as compared with $\mathrm{Sgo}^{-/-}$spermatocytes (Extended Data Fig. 6b), indicating that the protection defects caused by $\mathrm{Meikin}^{-/-}$are somewhat weaker than those by $\mathrm{Sgo}^{-/-}$.

\section{MEIKIN facilitates mono-orientation}

Because meiosis I chromosome alignment is partly perturbed in Meikin ${ }^{-/-}$oocytes (Fig. 2b), we postulated that mono-orientation of sister kinetochores might be also impaired in the mutant mice as it is in fission yeast moals cells ${ }^{23}$. Consistently, we found that the sister kinetochore distance of prometaphase I chromosomes is significantly increased $(\sim 20 \%)$ in Meikin $^{-1-}$ mice as compared to wild-type mice (Fig. 3a, Extended Data Fig. 6c). Given that mono-orientation defects are significantly suppressed by chiasmata (or tension between homologs $)^{23,26}$, we sought to use $M l h 1^{-/-}$mice that have severely reduced chiasmata and accumulate univalent chromosomes in meiosis I. Because of the intrinsic property of mono-orientation of sister kinetochores, most univalents in $\mathrm{MlhI}^{-/-}$oocytes fail to align on the metaphase plate and largely inhibit the onset of anaphase $\mathrm{I}^{27}$ (Fig. 3b). Interestingly, further depletion of MEIKIN (MlhI ${ }^{-/} \mathrm{Meikin}^{-/}$) alleviates the anaphase I inhibition, although anaphase I onset is delayed in $\mathrm{Meikin}^{-/-}$when chiasmata are present (Fig. 2a, 3b). We examined chromosome alignment at $10 \mathrm{~h}$ after GVBD when most $M l h 1^{-/-}$or $M l h 1^{-/}$ Meikin $^{-/}$oocytes have not yet entered anaphase I. Univalents congressed at the metaphase plate and sister kinetochore pairs separated by $>0.6 \mu \mathrm{m}$ were seen more frequently in $\mathrm{Mlh1}^{-/-}$Meikin $^{-/-}$than $\mathrm{Mlh1}^{-/-}$(Fig. 3c). Thus we infer that mono-orientation is impaired in $\mathrm{Mlhl}^{\text {- }^{-}} \mathrm{Meikin}^{-/-}$oocytes. In contrast, $\mathrm{Mlhl}^{-/-} \mathrm{Sgo}^{-/-}$oocytes did not show such defects (Fig. 3c). Similar mono-orientation defect was observed in $\mathrm{Mlhl}^{-1-} \mathrm{Meikin}^{-/-}$spermatocytes (Fig. 3d). Therefore, we conclude that MEIKIN plays a crucial role in mono-orientation in addition to its role in protecting centromeric cohesion.

\section{MEIKIN recruits PLK1}

To elucidate the molecular function of MEIKIN, we searched for MEIKIN-interacting proteins in a yeast two-hybrid system as well as by immunoprecipitation-MASS spectrometry. In both assays, we obtained Polo-like kinase PLK1 in addition to CENP-C (Extended Data Fig. 7). Immunoprecipitation assays indicated that MEIKIN co-precipitates PLK1 in testis chromatin extracts (Fig. 4a). Immunostaining using spermatocytes indicated that PLK1 localizes at kinetochores in diplotene, culminates in metaphase I and declines during anaphase I when MEIKIN disappears completely. In metaphase II, centrosome localization is prominent but kinetochore localization is markedly decreased to levels comparable to those in mitosis, indicating that the kinetochore enrichment of PLK1 is specific to meiosis I (Fig. 4b). 
Strikingly, PLK1 signals at kinetochores were diminished in Meikin ${ }^{-/-}$oocytes and largely dispersed in Meikin $^{-/-}$spermatocytes (Fig. 4b, c). These results indicate that MEIKIN plays a crucial role in enriching PLK1 to kinetochores in diplotene and in sustaining its localization at least until anaphase I.

\section{PLK1 acts in protection and mono-orientation}

The foregoing results suggest the possibility that PLK1 activity enriched to kinetochores might be required for kinetochore regulation in meiosis I. To explore this possibility, we sought to deplete PLK1 activity during meiosis I by adding the PLK1 inhibitor BI 2536 (ref. ${ }^{28}$ ) to the oocyte culture. We treated oocytes with BI 2536 for $2 \mathrm{~h}$ between $4 \mathrm{~h}$ and $6 \mathrm{~h}$ after GVBD, because this transient treatment allowed $\sim 70 \%$ of oocytes to undergo meiosis I division and suppressed PLK1 activity at kinetochores (Extended Data Fig. 8a, b). More than half of BI 2536-treated oocytes showed chromosome misalignment in metaphase II, most likely because partial or complete sister chromatid separation happen during anaphase I (Fig. 4d, Extended Data Fig. 8c). Accordingly, centromeric REC8 on metaphase II chromosomes was largely diminished in the BI 2536-treated oocytes (Fig. 4e, Extended Data Fig. 8d). These results suggest that PLK1 activity is required, at least after prometaphase I, for the protection of centromeric REC8 cohesin in the following anaphase I.

We next examined whether PLK1 activity is also required for the mono-orientation of sister kinetochores in meiosis I by treating $M l h I^{-/-}$oocytes with BI 2536 between $9 \mathrm{~h}$ and $10 \mathrm{~h}$ after GVBD. While univalents largely failed to congress in $M l h 1^{-/-}$oocytes, BI 2536 treatment significantly increased the numbers of univalents congressed at the metaphase plate and sister kinetochore pairs separated by $>0.6 \mu \mathrm{m}$ (Fig. 4f, Extended Data Fig. 8e). Thus, we conclude that PLK1 also plays a role in promoting mono-orientation in oocytes.

\section{Conservation of MEIKIN}

The foregoing analyses suggest that mouse MEIKIN is analogous to $S$. pombe Moal because both mutants show defects in mono-orientation and the protection of centromeric cohesion, albeit to different extents ${ }^{23}$. Accordingly, we have found that, like MEIKIN, Moal carries a putative polo-box domain (PBD)-binding site (Ser-Thr-Pro) ${ }^{29}$ (Fig. 5a). Immunoprecipitation assays revealed that Moa1 associates with the fission yeast Polo-like kinase Plo1 through the PBD-binding motif in vivo (Fig. 5b). Because Plol is indeed localized to kinetochores in meiosis $\mathrm{I}^{30}$ (Fig. 5c), we examined the functional relationship between Plo1 and Moa1. Meiosis I-specific enrichment of Plo1 to kinetochores was abolished in cells carrying a mutation in the PBD-binding motif of Moal (moal-101A) as well as in moals cells (Fig. 5c). 
We then examined the effects of these mutations on meiotic chromosome segregation in recombination-deficient ( rec 12 $\Delta$ ) zygotes, in which chiasmata are not formed. The results indicate that moal-101A cells undergo equational segregation of sister chromatids in meiosis I, as in moal $\Delta$ cells (Fig. 5d), stressing the importance of the PBD-binding motif for Moal function.

To delineate the importance of Plo1 over Moa1, we sought to inactivate Plo1 in meiosis. Because a reduction in Plol at the cellular level impairs spindle formation and nuclear division (data not shown), we sought to mutate endogenous Plo1 to become cleavable by tobacco etch virus (TEV) protease and thus inactive only around the kinetochore by co-expressing Cnp3C-TEV (TEV protease fused with a kinetochore-localizing peptide) ${ }^{23}$. Indeed, plo1-tev cells expressing Cnp3C-TEV showed a reduction of Plo1-tev signals selectively from kinetochores with preserving the localization at SPBs (yeast centrosomes) in metaphase I, whereas plol-tev cells expressing a control Cnp3C showed normal localization of Plo1-tev (Fig. 5e). Strikingly, plo1-tev cells expressing Cnp3C-TEV largely underwent equational segregation at meiosis I (Fig. 5f). Thus, we conclude that Plo1 recruited by Moa1 to kinetochores fulfils a crucial role in promoting mono-orientation and centromeric cohesion protection in fission yeast as in mice.

The foregoing results recalled the fact that budding yeast Spo13 associates and cooperates with PLK $(\mathrm{Cdc} 5)^{31}$, although there is no apparent amino acid sequence homology between Moa1 and Spo13, and a meiotic role for Cdc5 in the protection of cohesin is a subject of debate $^{16,32,33}$ (Fig. 5a). To examine the functional conservation between Spo13 and Moa1, we expressed Spo13 fused with the kinetochore targeting peptide Cnp3C in moals cells (note that Spo13 itself cannot localize to fission yeast kinetochores; data not shown). Strikingly, the mono-orientation defect of moal $\Delta$ was significantly suppressed by Spo13-Cnp3C, but not by Spo13-145A-Cnp3C, which has a mutation in the PBD-binding motif ${ }^{31}$ (Fig. 5g). Similar results were obtained by expressing Moa1 and its mutant version fused to $\mathrm{Cnp} 3 \mathrm{C}$ in moals cells, although Moa1-Cnp3C produced a more robust suppression. These results strongly suggest that Spo13 is the functional homolog of Moal.

\section{Discussion}

Motivated by our previous finding that $S$. pombe meiotic kinetochore regulator Moal binds to CENP-C ${ }^{24}$, we have identified a meiosis-specific kinetochore protein MEIKIN in mice. Although there is no significant sequence homology between MEIKIN and Moa1 ${ }^{23}$, our study reveals striking biochemical and functional similarities between these two factors. Firstly, 
MEIKIN and Moa1 localize to kinetochores only in meiosis I through their C-terminus domains, which bind directly to CENP-C, but never appear in meiosis II or mitosis. Secondly, MEIKIN and Moa1 play a crucial role in promoting mono-orientation and protecting centromeric cohesion. Thirdly, MEIKIN and Moal both recruit PLK to kinetochores and PLK kinase activity is required for their functions. Based on this evidence, we conclude that MEIKIN and Moa1 are functional homologs. Further, it turns out that these proteins share some properties with budding yeast Spo13, which is localized to meiotic kinetochores in a meiosis I-specific manner and required for mono-orientation and centromeric cohesion protection $^{21}, 22,26,31,34$. Our functional assays in fission yeast demonstrated that centromere-tethered Spo13 suppresses the moal defects depending on its PLK binding. These results are consistent with and support the notion that budding yeast Cdc5 is required for mono-orientation and for protecting cohesin at centromeres during meiosis $\mathrm{I}^{16,32}$. Taken together, our results identify MEIKIN, Moal and Spo13 as functional homologs that form a novel protein family, for which we propose the name 'meikin' (Fig. 5h).

Although studies in budding yeast identified monopolin, which acts downstream of Spo13 to assemble a single kinetochore form one pair of centromeres ${ }^{20-22,35-37}$, a fission yeast monopolin homolog is dispensable for mono-orientation ${ }^{38,39}$. Instead, the fission yeast cohesin Rec8 plays a central role in mono-orientation downstream of Moa1 by establishing cohesion at the core centromeres and thereby conjoining sister kinetochores ${ }^{23,40}$, a mechanism that might be lacking in budding yeast ${ }^{20,35}$ (Fig. 5h). In mouse oocytes as in fission yeast, the artificial cleavage of REC8 cohesin at the core centromeres disrupts mono-orientation ${ }^{23,41}$, implying that REC8 is acting for mono-orientation downstream of meikin and this might be conserved in plants as well ${ }^{9}$ (Fig. 5h). As fission yeast Moal has been shown to be required only after DNA replication ${ }^{42}$, MEIKIN starts to localize at kinetochores in the pachytene stage so that MEIKIN may also regulate the maintenance of cohesion rather than its establishment. Consistent with these results, our analyses show that sister kinetochores on prometaphase I chromosomes are conjoined in wild-type mice but split in Meikin ${ }^{-/-}$mice, although they do not separate so much as compared with mitotic prometaphase chromosomes (Fig. 3a, Extended Data Fig. 6c). Accordingly, the mono-orientation defects are mild so that they can be detected in univalents in Meikin ${ }^{-/}$ $\mathrm{Mlh1}^{-/-}$mice but little in Meikin ${ }^{-/}$bivalents (Fig. 2b, 3c). We therefore assume that in addition to sister-kinetochore association promoted by meikin, meiotic pericentric chromatin including REC8 cohesin may also structurally contribute to restrain sister kinetochore separation, and thereby to bias to mono-orientation. 
We showed that MEIKIN promotes the protection of centromeric cohesion at least partly by stabilizing $\mathrm{SGO} 2$ at the centromeres, and that the defects in cohesion protection are somewhat weaker in $\mathrm{Meikin}^{-/}$mice as compared with $\mathrm{Sgo2}^{-/-}$mice. Thus, the protection of centromeric cohesion is not entirely abolished in $\mathrm{Meikin}^{-/}$mice, and intriguingly this is also the case in fission yeast moal $\Delta$ and budding yeast $s p o 13 \Delta$ cells $^{21,23}$. These results suggest that meikin might be a regulator of shugoshin-dependent protection pathway rather than a bona fide protector of cohesin (Fig. 5h). Although meikin acts to enrich PLK to kinetochores, meikin might not be a mere recruiter of PLK. In fact, PLK is localized to kinetochores in mitotic cells ${ }^{43,44}$ albeit less so than in meiotic cells, and PLK reduction is mild in Meikin ${ }^{-/-}$ oocytes compared to spermatocytes, although meiotic kinetochore functions are similarly defective in female and male. Therefore, we assume that meikin may play additional roles such as in PLK substrate recognition and have some sort of regulatory function. In sum, our studies in mouse together with those in yeasts provide a unified model, in which the conserved key regulator meikin cooperates with PLK to promote mono-orientation and centromeric cohesion protection, two hallmarks of meiotic kinetochore function in eukaryotes. The current study thus provides a clear answer to the longstanding question of how these two distinct kinetochore functions, both essential for meiosis I, are coordinated at the molecular levels.

Accumulating evidence suggests that bivalents in aged mouse oocytes show defects in sister chromatid cohesion (or REC8 maintenance), which would increase chromosome segregation errors in the following meiotic divisions ${ }^{45-48}$. Our study revealed that MEIKIN localizes to kinetochores already in prophase I and probably functions beyond several months until ovulation in mouse (Extended Data Fig. 3b), a property similar to that of REC8 cohesin. Because MEIKIN is conserved in humans (Extended Data Fig. 9), MEIKIN might be a candidate influencing on age-associated chromosome segregation errors, a major cause of human birth defects ${ }^{49}$.

1. Uhlmann, F., Lottspeich, F. \& Nasmyth, K. Sister-chromatid separation at anaphase onset is promoted by cleavage of the cohesin subunit Scc1. Nature 400, 37-42 (1999).

2. Peters, J.M., Tedeschi, A. \& Schmitz, J. The cohesin complex and its roles in chromosome biology. Genes Dev 22, 3089-3114 (2008).

3. Nasmyth, K. \& Haering, C.H. Cohesin: its roles and mechanisms. Annu Rev Genet 43, 525-558 (2009).

4. Buonomo, S.B. et al. Disjunction of homologous chromosomes in meiosis I depends 
on proteolytic cleavage of the meiotic cohesin Rec8 by separin. Cell 103, 387-398. (2000).

5. Kitajima, T.S., Miyazaki, Y., Yamamoto, M. \& Watanabe, Y. Rec8 cleavage by separase is required for meiotic nuclear divisions in fission yeast. Embo $J \mathbf{2 2}$, 5643-5653 (2003).

6. Tachibana-Konwalski, K. et al. Rec8-containing cohesin maintains bivalents without turnover during the growing phase of mouse oocytes. Genes Dev 24, 2505-2516 (2010).

7. Moore, D.P. \& Orr-Weaver, T.L. Chromosome segregation during meiosis: building an unambivalent bivalent. Curr Top Dev Biol 37, 263-299 (1998).

8. Brar, G.A. \& Amon, A. Emerging roles for centromeres in meiosis I chromosome segregation. Nat Rev Genet 9, 899-910 (2008).

9. Watanabe, Y. Geometry and force behind kinetochore orientation: lessons from meiosis. Nat Rev Mol Cell Biol 13, 370-382 (2012).

10. Kitajima, T.S., Kawashima, S.A. \& Watanabe, Y. The conserved kinetochore protein shugoshin protects centromeric cohesion during meiosis. Nature 427, 510-517 (2004).

11. Lee, J. et al. Unified mode of centromeric protection by shugoshin in mammalian oocytes and somatic cells. Nature Cell Biol. 10, 42-52 (2008).

12. Llano, E. et al. Shugoshin-2 is essential for the completion of meiosis but not for mitotic cell division in mice. Genes Dev 22, 2400-2413 (2008).

13. Kitajima, T.S. et al. Shugoshin collaborates with protein phosphatase $2 \mathrm{~A}$ to protect cohesin. Nature 441, 46-52 (2006).

14. Riedel, C.G. et al. Protein phosphatase 2A protects centromeric sister chromatid cohesion during meiosis I. Nature 441, 53-61 (2006).

15. Marston, A.L., Tham, W.H., Shah, H. \& Amon, A. A genome-wide screen identifies genes required for centromeric cohesion. Science 303, 1367-1370 (2004).

16. Katis, V.L. et al. Rec8 phosphorylation by casein kinase 1 and Cdc7-Dbf4 kinase regulates cohesin cleavage by separase during meiosis. Dev Cell 18, 397-409 (2010).

17. Ishiguro, T., Tanaka, K., Sakuno, T. \& Watanabe, Y. Shugoshin-PP2A counteracts casein-kinase-1-dependent cleavage of Rec8 by separase. Nat Cell Biol 12, 500-506 (2010).

18. Hugerat, Y. \& Simchen, G. Mixed segregation and recombination of chromosomes and YACs during single-division meiosis in spo13 strains of Saccharomyces cerevisiae. Genetics 135, 297-308 (1993).

19. Klapholz, S. \& Esposito, R.E. Recombination and chromosome segregation during the single division meiosis in SPO12-1 and SPO13-1 diploids. Genetics 96, 589-611. 
(1980).

20. Toth, A. et al. Functional genomics identifies monopolin: a kinetochore protein required for segregation of homologs during meiosis I. Cell 103, 1155-1168 (2000).

21. Katis, V.L. et al. Spo13 facilitates monopolin recruitment to kinetochores and regulates maintenance of centromeric cohesion during yeast meiosis. Curr. Biol. 14, 2183-2196 (2004).

22. Lee, B.H., Kiburz, B.M. \& Amon, A. Spo13 maintains centromeric cohesion and kinetochore coorientation during meiosis I. Curr. Biol. 14, 2168-2182 (2004).

23. Yokobayashi, S. \& Watanabe, Y. The kinetochore protein Moal enables cohesion-mediated monopolar attachment at meiosis I. Cell 123, 803-817 (2005).

24. Tanaka, K., Chang, H.L., Kagami, A. \& Watanabe, Y. CENP-C functions as a scaffold for effectors with essential kinetochore functions in mitosis and meiosis. Dev Cell 17, 334-343 (2009).

25. Kitajima, T.S., Ohsugi, M. \& Ellenberg, J. Complete kinetochore tracking reveals error-prone homologous chromosome biorientation in mammalian oocytes. Cell 146, 568-581 (2011).

26. Shonn, M.A., McCarroll, R. \& Murray, A.W. Spo13 protects meiotic cohesin at centromeres in meiosis I. Genes Dev 16, 1659-1671. (2002).

27. Woods, L.M. et al. Chromosomal influence on meiotic spindle assembly: abnormal meiosis I in female Mlh1 mutant mice. J Cell Biol 145, 1395-1406 (1999).

28. Lenart, P. et al. The small-molecule inhibitor BI 2536 reveals novel insights into mitotic roles of polo-like kinase 1. Curr Biol 17, 304-315 (2007).

29. Elia, A.E. et al. The molecular basis for phosphodependent substrate targeting and regulation of Plks by the Polo-box domain. Cell 115, 83-95 (2003).

30. Krapp, A., Del Rosario, E.C. \& Simanis, V. The role of Schizosaccharomyces pombe dma1 in spore formation during meiosis. J Cell Sci 123, 3284-3293 (2010).

31. Matos, J. et al. Dbf4-dependent CDC7 kinase links DNA replication to the segregation of homologous chromosomes in meiosis I. Cell 135, 662-678 (2008).

32. Clyne, R.K. et al. Polo-like kinase Cdc5 promotes chiasmata formation and cosegregation of sister centromeres at meiosis I. Nature Cell Biol. 5, 480-485 (2003).

33. Attner, M.A., Miller, M.P., Ee, L.S., Elkin, S.K. \& Amon, A. Polo kinase Cdc5 is a central regulator of meiosis I. Proc Natl Acad Sci U S A 110, 14278-14283 (2013).

34. Lee, B.H., Amon, A. \& Prinz, S. Spo13 regulates cohesin cleavage. Genes Dev. 16, 1672-1681. (2002).

35. Monje-Casas, F., Prabhu, V.R., Lee, B.H., Boselli, M. \& Amon, A. Kinetochore orientation during meiosis is controlled by Aurora B and the monopolin complex. Cell 
128, 477-490 (2007).

36. Corbett, K.D. et al. The monopolin complex crosslinks kinetochore components to regulate chromosome-microtubule attachments. Cell 142, 556-567 (2010).

37. Sarangapani, K.K. et al. Sister kinetochores are mechanically fused during meiosis I in yeast. Science 346, 248-251 (2014).

38. Gregan, J. et al. The kinetochore proteins Pcs1 and Mde4 and heterochromatin are required to prevent merotelic orientation. Curr Biol 17, 1190-1200 (2007).

39. Tada, K., Susumu, H., Sakuno, T. \& Watanabe, Y. Condensin association with histone H2A shapes mitotic chromosomes. Nature 474, 477-483 (2011).

40. Sakuno, T., Tada, K. \& Watanabe, Y. Kinetochore geometry defined by cohesion within the centromere. Nature 458, 852-858 (2009).

41. Tachibana-Konwalski, K. et al. Spindle assembly checkpoint of oocytes depends on a kinetochore structure determined by cohesin in meiosis I. Curr Biol 23, 2534-2539 (2013).

42. Kagami, A. et al. Acetylation regulates monopolar attachment at multiple levels during meiosis I in fission yeast. EMBO Rep 12, 1189-1195 (2011).

43. Ahonen, L.J. et al. Polo-like kinase 1 creates the tension-sensing $3 \mathrm{~F} 3 / 2$ phosphoepitope and modulates the association of spindle-checkpoint proteins at kinetochores. Curr Biol 15, 1078-1089 (2005).

44. Kang, Y.H. et al. Self-regulated Plk1 recruitment to kinetochores by the Plk1-PBIP1 interaction is critical for proper chromosome segregation. Mol Cell 24, 409-422 (2006).

45. Hodges, C.A., Revenkova, E., Jessberger, R., Hassold, T.J. \& Hunt, P.A. SMC1beta-deficient female mice provide evidence that cohesins are a missing link in age-related nondisjunction. Nat Genet 37, 1351-1355 (2005).

46. Lister, L.M. et al. Age-related meiotic segregation errors in mammalian oocytes are preceded by depletion of cohesin and Sgo2. Curr Biol 20, 1511-1521 (2010).

47. Chiang, T., Duncan, F.E., Schindler, K., Schultz, R.M. \& Lampson, M.A. Evidence that weakened centromere cohesion is a leading cause of age-related aneuploidy in oocytes. Curr Biol 20, 1522-1528 (2010).

48. Jessberger, R. Age-related aneuploidy through cohesion exhaustion. EMBO Rep 13, 539-546 (2012).

49. Hassold, T. \& Hunt, P. To err (meiotically) is human: the genesis of human aneuploidy. Nat Rev Genet 2, 280-291 (2001).

50. Baker, S.M. et al. Involvement of mouse Mlh1 in DNA mismatch repair and meiotic crossing over. Nat Genet 13, 336-342 (1996). 


\section{Acknowledgements}

We thank K. Tachibana-Konwalski for the C57BL/6 Mlh $1^{\text {tmlLiskay }}-\mathrm{KO}$ mice and and J. Ellenberg for a macro for automated microscopy. We also thank J. Lee, T. Hirano, S. Fujiyama, Y. Yamazumi, as well as the Gotoh laboratory for technical advice and all members of the Watanabe laboratory for their support and discussion. This work was supported in part by a JSPS Research Fellowship (to J.K.), SAF2011-25252 (to A.M.P.), a research grant from Uehara Memorial Foundation, a Grant-in-Aid for Young Scientists (B) (to T.S.K.), a Grant-in-Aid for Scientific Research on Innovative Areas, a Grant-in-Aid for Scientific Research (C) (to K.I.), and a Grant-in-Aid for Specially Promoted Research (to Y.W.) from MEXT, Japan.

\section{Author Contributions}

J.K., supported by K.I. performed most of the experiments in mice. K.I. and N.T. generated Meikin-knockout mice. A.M.P. provided Sgo2-knockout mice. A.N. isolated MEIKIN in yeast two-hybrid screening. B.A., S.Y., A.K., T.I. and T.S. performed experiments in fission yeast. Y.S. and T.S.K. performed live imaging. The experimental design and interpretation of data were conducted by J.K., K.I., B.A., S.Y., A.K., T.I. T.S.K., Y.T. and T.S. Y.W. supervised the project, and wrote the paper with input from all authors.

\section{Author information}

Sequence data are deposited with NCBI GenBank under accession numbers AB987828 for mMEIKIN and AB987829 for hMEIKIN. Reprints and permissions information is available at www.nature.com/reprints. The authors declare no competing financial interests. Readers are welcome to comment on the online version of this paper. Correspondence and requests for materials should be addressed to Y.W. (ywatanab@iam.u-tokyo.ac.jp).

\section{METHODS}

\section{Yeast two-hybrid screening and assay}

For yeast two-hybrid screening, mouse CENP-C cDNA encoding the C-terminus (a.a. 692-906) and mouse Meikin cDNA encoding the C-terminus (a.a. 272-434) were subcloned into the vector pGBKT7. The bait strains were raised by transforming the bait vector into the yeast strain AH109. A matchmaker mouse testis cDNA library (Clontech) was transformed into the bait strains, and positive transformants were selected on nutrition-restricted plates 
(SD-trp-leu-his-ade, $+10 \mathrm{mM}$ 3AT). Positive transformants were further examined by blue/white assay to confirm the interaction. Prey plasmids were extracted from the candidate clones and sequenced. To exclude false positive clones, the candidate prey plasmids were retransformed into the yeast strain AH109 along with the bait vector or negative control bait vector of $\mathrm{p} 53$.

For yeast two-hybrid assays, the following cDNAs were subcloned into the bait vector pGBKT7: hCENP-C full length, hCENP-C C-terminal region (a.a. 732-945), mPLK1 full length, hPLK1 full length. The following cDNAs were subcloned into prey vector pACT2 : MEIKIN full length, MEIKIN N-terminus (a.a. 1-271), MEIKIN C-terminus (a.a. 272-434), MEIKIN Ex1-12 (a.a. 1-384), MEIKIN Ex 13-14 (a.a. 385-434). The following cDNAs were subcloned into the prey vector pGADT7 : hMEIKIN N-terminus (a.a. 1-264), hMEIKIN C-terminus (a.a. 259-373). These bait and prey preparations were co-transformed into the yeast strain AH109.

\section{Mice}

Sgo2- and Mlh1 $1^{\text {tmlLiskay }}$ - knockout mice were reported earlier ${ }^{12,50}$. All single and double knockout mice were congenic with the C57BL/6 background. Whenever possible, each knockout animal was compared to littermates or age-matched non-littermates from the same colony, unless otherwise described. Animal experiments were approved by the Institutional Animal Care and Use Committee (approval \#23001, \#23013, \#24001, \#25012) at IMCB and AH23-05-04 at CDB).

\section{Generation of Meikin (4930404A10rik) knockout mouse and genotyping}

The targeting vector was designed to disrupt Exon 4 of the Meikin genomic locus. Targeting arms of $4.40 \mathrm{~kb}$ and $4.98 \mathrm{~kb}$ fragments, 5' and 3' to the Exon 4 of Meikin gene respectively, were generated by PCR from mouse C57BL/6 BAC clone (RPCI23-32I13) and directionally cloned flanking $\mathrm{p} G K$-Neo-polyA and $D T-A$ cassettes. The homologous recombinant cells were isolated using Baltha1 ES cells derived from $\mathrm{C} 57 \mathrm{BL} / 6 \mathrm{~N}^{51}$, and chimeric mice were generated by morula injection (host ICR) of recombinant ES cells (Kumamoto university, CARD). The G418-resistant ES clones were screened for homologous recombination with the Meikin locus by PCR using primers:

MEIKIN-7547F : 5'-GTTCAGTTTCACCTCCCGGTCTGAC and

Neo3R: 5'-TACCGGTGGATGTGGAATGTGTGC for the left arm (4734 bp),

Neo104F : 5'-ggaccgctatcaggacatagcgttgge and

MEIKIN-18860R: 5'-ACTCGCCACTGACTTCTCCTGTGAGC for the right arm (5531bp). 
Southern blot was used to confirm correctly targeted ES cell clones: DNA was digested with Pvu II for Southern blots with an external 5'-probe. Chimeric males were mated to C57BL/6 females and the progeny were genotyped by PCR using the following primers. Ex3F (common-forward): 5'- CCCCAGAGGAAAAGACACCACC-3', Ex4R (wild-type-reverse): 5'-CTCGACAACAAGCTGTCCATCTC-3' and Neo4R (mutant-reverse): 5'-CATGAGTGGGAGGAATGAGCTGGC-3' for the Meikin mutant allele (3473 bp) and the wild-type allele (1750 bp).

\section{Histological analyses}

The testes, epididymides or ovaries from mice (12-week-old males, 8 -week-old in female) were fixed in Bouin's solution and embedded in paraffin. Sections were prepared on APS-coated slides (Matsunami) at $5 \mu \mathrm{m}$ thickness. The slides were dehydrated and stained with hematoxylin and eosin.

\section{Antibody production}

Polyclonal antibodies against mouse MEIKIN (a.a. 1-434) and MEIKIN C-terminal (a.a. 317-434) were generated by immunizing rabbits and mice. Polyclonal antibodies against human MEIKIN N- and C-terminal regions were generated by immunizing ICR mice. All His-tagged recombinant proteins were produced by inserting cDNA fragments in-frame with $\mathrm{pET} 19 \mathrm{~b}$ or pET28c (Novagen). All His-tagged recombinant proteins were purified by Ni-NTA (QIAGEN) under denaturing conditions using $6 \mathrm{M} \mathrm{HCl-Guanidine.} \mathrm{The} \mathrm{antibodies}$ were affinity-purified from the immunized serum with immobilized peptides on CNBr-activated Sepharose (GE healthcare).

\section{Antibodies}

The following primary antibodies were used for immunoblot (IB) and immunofluorescence (IF) studies: mouse anti-tubulin (IB, 1:5000, IF, 1:1000, DM1A, Sigma, Cat\#T9026), human ACA (IF, 1:20, MBL, Cat\#NA-8184), mouse anti-PLK1 (IB and IF, 1:1000, Abcam, Cat\#ab17056), rabbit anti-CENP-U/MLF1 phosphoT78 (IF, 1:100, Abcam, Cat\#ab34911), rabbit anti-Histone H3 (IB, 1:1000, Abcam, Cat\#ab1791), rabbit anti-GFP (IF, 1:1000, Invitrogen, Cat\#A11122). The following polyclonal antibodies were described previously: rabbit anti-mCENP-C (IB and IF, 1:1000), rabbit anti-mREC8 (IF, 1:500), mouse anti-mREC8 (IF, 1:500), mouse anti-mSYCP3 (IF, 1:1000) ${ }^{52}$. Mouse anti-mSGO2 (IF, $1: 500)^{53}$. Rabbit anti-mSGO2 (IF, 1:1000) ${ }^{11}$. Rat anti-mSYCP3 polyclonal antibody (IF, $1: 1000)^{54}$. 
The following secondary antibodies were used for immunofluorescence studies: Alexa488-conjugated donkey anti-mouse (Invitrogen, Cat\#A21202), donkey anti-rabbit (Invitrogen, Cat\#A21206), Alexa555-conjugated goat anti-mouse (Invitrogen, Cat\#A21422), donkey anti-rabbit (Invitrogen, Cat\#A31572), goat anti-human (Invitrogen, Cat\#A21433), goat anti-rat (Invitrogen, Cat\#A21434), Alexa647-conjugated donkey anti-mouse (Invitrogen, Cat\#A31571), goat anti-rabbit (Invitrogen, Cat\#A21244), goat anti-human (Invitrogen, Cat\#A21445), goat anti-rat (Invitrogen, Cat\#A21247).

\section{In vitro oocyte culture}

Ovaries collected from 6 to 12 -week-old female mice were used for this study after 46 to $48 \mathrm{~h}$ of treatment with 5 IU of pregnant mare serum gonadotropin. GV oocytes were isolated by puncturing the follicles in M2 medium (Sigma) containing $250 \mu \mathrm{M}$ 3-isobutyl-1-metyl-xanthine (IBMX, Sigma) to maintain prophase arrest. To induce resumption of meiosis, the oocytes were cultured in M16 medium (Sigma) supplemented with $10 \%$ FBS in a $5 \% \mathrm{CO}_{2}$ atmosphere at $37^{\circ} \mathrm{C}$. Oocytes that had not undergone $\mathrm{GV}$ breakdown (GVBD) by $90 \mathrm{~min}$ were removed from the experiment. For oocyte drug treatment, $100 \mathrm{nM}$ BI 2536 (ChemieTek), $5 \mu \mathrm{M}$ reversine (Sigma) and $10 \mu \mathrm{M}$ nocodazole (Sigma) were added to the culture medium at the indicated time point; control oocytes were treated with an equivalent volume of DMSO.

\section{Immunofluorescence microscopy of spermatocytes and oocytes}

Squashed spermatocytes were prepared from adult male mice as described previously ${ }^{12}$ with modification. Briefly, seminiferous tubules were minced, and fixed in fixation buffer of $2 \%$ paraformaldehyde (PFA)/0.1\% Triton X-100/PBS. The cell suspension was filtered through a cell strainer (BD Falcon) to remove debris, pipetted repeatedly and centrifuged. The cell pellets were suspended in the fixation buffer and gently squashed by the cover glass. After fixation, the slides were frozen in liquid nitrogen. For immunostaining, the frozen slides were immersed in PBS and the coverslips were removed.

Fixed whole mount oocytes was prepared as described previously ${ }^{11,25}$ with minor modification. After in vitro culture, the oocytes were fixed in the fixation buffer for $30 \mathrm{~min}$ at room temperature, washed with PBS and then blocked with 3\% BSA/PBT $(0.1 \%$ Triton $\mathrm{X}-100 / \mathrm{PBS}$ ) for $1 \mathrm{~h}$ at room temperature. For measurement of sister kinetochore distance at early prometaphase I, oocytes were exposed to $1 \%$ Pronase (Sigma) to remove the zona pellucida, and fixed in the fixation buffer on glass slides for $3 \mathrm{~h}$. 
Oocyte chromosome spreads were prepared as described previously ${ }^{55}$. Briefly, after zona pellucida removal, oocytes were transferred onto glass slides and fixed in a solution of $1 \%$ PFA/0.15\% Triton $\mathrm{X}-100$ /distilled $\mathrm{H}_{2} \mathrm{O}$ adjusted to $\mathrm{pH}$ 9.2. After a quick dry, chromosomes were immunostained as described above. Fetal oocyte chromosome spreads were prepared as described previously ${ }^{56}$.

For immunostaining spermatocytes and oocytes, samples were briefly washed in PBS, blocked with 3\% BSA/PBS for $10 \mathrm{~min}$ at room temperature and incubated with primary antibodies in 3\% BSA/PBS for $1 \mathrm{~h}$ and secondary antibodies for $1 \mathrm{~h}$ at room temperature. In whole mount oocytes, incubation was performed at $4{ }^{\circ} \mathrm{C}$ for overnight for each antibody. The slides were washed with PBS, and mounted using VECTASHIELD medium with DAPI (Vector Laboratories).

Images were acquired on an IX-70 microscope (Olympus) equipped with a CoolSnap HQ CCD camera (Roper Scientific), DeltaVision Core system (GE Healthcare). Only whole mount oocytes images were captured with an FV1000 confocal laser scanning microscope at $1 \mu \mathrm{m}$ intervals and processed with FLUOVIEW software (Olympus).

The projection of the images, the quantification of signal intensity and the measurement of sister kinetochore distance were carried out with the SoftWorx software program (GE Healthcare). For the measurement of sister kinetochore distance, images were acquired with $\mathrm{Z}$-sections encompassing the entire nuclei. The peak-to-peak distance of CENP-C signals was measured for a pair of sister kinetochores in structurally preserved nuclei. The sister kinetochores distance was measured by calculating the square root of $X^{2}+Y^{2}+Z^{2}$.

\section{Exogenous expression of GFP-tagged MEIKIN variants in testis}

For exogenous expression of GFP-tagged MEIKIN variants in testis, plasmid DNA was injected into live-mouse testes as described previously ${ }^{57}$. Meikin variants were cloned in pCAG vector. Plasmid DNA was injected into live-mouse testes as described previously ${ }^{57}$. Briefly, male mice at 16-20 days postpartum were anesthetized with pentobarbital and the testes were pulled from the abdominal cavity. $50 \mu \mathrm{g}$ of plasmid DNA $(10 \mu \mathrm{l}$ of $5 \mu \mathrm{g} / \mu \mathrm{l}$ DNA solution) was injected into each testis using glass capillaries under a stereomicroscope (M165C; Leica). Testes were held between a pair of tweezer-type electrodes (CUY21; BEX), and electric pulses were applied four times and again four times in the reverse direction at 30 $\mathrm{V}$ for $50 \mathrm{~ms}$ for each pulse. The testis was then returned to the abdominal cavity, and the abdominal wall and skin were closed with sutures. Immunostaining was performed 24-48 $\mathrm{h}$ 
after electroporation to assess the localization of the GFP-tagged Meikin variants in spermatocyte using rabbit anti-GFP (Invitrogen). Because the efficiency of GFP-tagged protein expression in meiotic prophase is $5-10 \%$, we detected kinetochore localization of GFP-tagged MEIKIN from GFP-positive cells.

\section{Live confocal imaging of oocytes}

Oocytes were microinjected with in vitro-transcribed RNAs encoding 2 mEGFP-CENP-C (0.8 $\mathrm{pg}$ ) and H2B-mCherry (0.2 pg), and incubated for $3 \mathrm{~h}$ before IBMX washout. Timing of GVBD was determined by low resolution time-lapse imaging. Oocytes not undergoing GVBD within $1.5 \mathrm{~h}$ after the IBMX washout were removed from further imaging analysis. High resolution imaging was started around $5.5 \mathrm{~h}$ after GVBD. Imaging was performed with Zeiss LSM710 equipped with a 40x C-Apochromat 1.2 W Corr M27 objective lens (Carl Zeiss) and a 3D multi-location tracking macro ${ }^{58}$. We acquired 17 z-confocal sections (every $1.5 \mu \mathrm{m}$ ) of 2-time-averaged 512 x 512 pixel xy images, which covered a volume of $30.36 \mu \mathrm{m}$ x $30.36 \mu \mathrm{m} \times 25.5 \mu \mathrm{m}$ at 5-min time intervals. 2mEGFP-CENP-C signals were peak-enhanced and background-subtracted as previously described ${ }^{25}$. The images were 3D-reconstructed with Imaris (Bitplane) and the kinetochore signals during anaphase were manually tracked. The kinetochore tracks were visualized by POV-Ray (www.povray.org).

\section{PCR with reverse transcription}

Total RNA was isolated from tissues using Trizol (Invitrogen). cDNA was generated from 0.5 $\mathrm{Mg}$ of total RNA using Superscript III (Invitrogen) followed by PCR amplification using Ex-Taq polymerase (Takara) and template cDNA (derived from 1ng RNA equivalent). Sequences of primers used to generate RT-PCR products from cDNA are as follows:

Meikin-F2 :5' -agatggacagcttgttgtcgagta-3';

Meikin k-R2 :5'-ctcagcaaatacaacctcagaagc-3';

GAPDH-F :5'-ttcaccaccatggagaaggc-3';

GAPDH-R :5'-ggcatggactgtggtcatga-3';

mSMC1-3516F:5' -ttacatcaaggaacagtcaacttgc-3';

mSMC1--3702R: 5'-ctattgttcattggggttggggttg-3';

Zfp438-F1:5' -gtatgcaaggacctgacaactcac-3';

Zfp438-R1:5' -catttgcttcctcctctctgtgta-3';

BC051142-F1:5' -ccttatcaaccttgtcagcettct-3';

BC051142-R1: 5' -ctcgaatctctttggacagcagta-3';

Nsun7-F1:5'-cetctcgatttaccatattctgcc-3';

Nsun7- R1: 5'-tatcaaagccttcacagagtggac-3'; 
Spesp1-F1:5' -catgaagctggtggtcctagttg-3';

Spesp1-R1:5' -gatggaccagaatgctgtactttg-3';

Snf1lk-F1:5' -gaggtccagctcatgaaacttttg-3';

Snf1lk-R1:5' -gctagcttgatatccatgttgctg-3';

Acbd3-F1:5' -caggagcagcactatcagcagtat

Acbd3-R1:5' -cgacttctcctcgacgtactgtaa

Ncor1-F1:5'-aactcttctggtggaggtgactct-3';

Ncor1-R1:5'-tgcccaggaataggagatttagac-3';

\section{Immunoprecipitation and mass spectrometry using testis extracts}

Testis chromatin-bound and -unbound extracts and immunoprecipitation were prepared as described previously ${ }^{52}$. Briefly, immunoprecipitations were performed with protein A-Dynabeads (Invitrogen)-conjugated rabbit anti-mCENP-C, rabbit anti-MEIKIN and control rabbit IgG (5 $\mu \mathrm{g}$ equivalent) from the chromatin-bound fraction prepared from $40-60$ wild-type testes (3-week-old). Co-immunoprecipitated proteins were run in 4-12\% NuPAGE gels (Invitrogen) in MOPS-SDS buffer and immunoblotted or analyzed by LC-MS/MS.

Fractions containing the MEIKIN immunoprecipitates were concentrated by precipitation with $10 \%$ trichloroacetic acid. The derived precipitates were dissolved in $7 \mathrm{M}$ Urea, $50 \mathrm{mM}$ Tris- $\mathrm{HCl}$ (pH 8.0), $5 \mathrm{mM}$ EDTA solution, with $5 \mathrm{mM}$ DTT at $37^{\circ} \mathrm{C}$ for $30 \mathrm{~min}$, and cysteine SH groups were alkylated with $10 \mathrm{mM}$ iodoacetamide at $37^{\circ} \mathrm{C}$ for $1 \mathrm{~h}$. After alkylation, the solutions were desalted by methanol/chloroform precipitation, and the precipitates were dissolved in $2 \mathrm{M}$ urea, $50 \mathrm{mM}$ Tris- $\mathrm{HCl}$ buffer and subjected to trypsin gold (Promega) digestion overnight at $37^{\circ} \mathrm{C}$. The resulting mixture of peptides was applied directly to the LC-MS/MS analysis system (Zaplous, AMR, Tokyo, Japan) using Finnigan LTQ mass spectrometry (Thermo Scientific) and a reverse phase C18 ESI column $(0.2 \times 50 \mathrm{~mm}$, LC assist). The protein annotation data were verified in the mouse NCBI sequences using Bioworks software (Ver. 3.3; Thermo Scientific) with quantitation featuring the SEQUEST search algorithm.

\section{Cloning of human Meikin homolog cDNA}

Human Meikin homolog cDNAs encoding the N-terminus (a.a. 1-264) and C-terminus (a.a. 259-373) were cloned from a human testis cDNA library (Takara) using the following primer sets :

NheI-hMEIKIN-N-1F :tACCGGT getagc atgtggecgetacgggtctatacce and NotI-hMEIKIN-N-795R: tACtCGGTgcggCCgCttattctgcttcaatacttgettttttc for N-terminus, 
NheI-hMEIKIN-C-1F: tACCGGTgctagcatgcagaaaacaaattccagtactc and

NotI-hMEIKIN-C-354R: tACtCGGTgcggCCgCtcatgccatttttattatgatatcttg for C-terminus.

\section{Immunostaining of human seminiferous tubule sections}

Frozen sections of adult human testis were purchased from Biochain. After washing with PBS/0.1\% Triton X100, immunostaining was performed as described above.

\section{Schizosaccharomyces pombe strain}

All strains used in this study are described in Supplementary Table 1. Complete medium (YE), minimal medium (SD and $\mathrm{MM}$ ) and sporulation-inducing medium (SPA) were used for

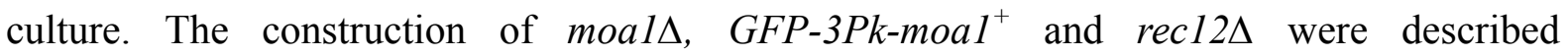
previously ${ }^{23}$. The C-terminal tagging of endogenous plo1 $^{+}$by GFP was performed using the PCR-based gene targeting method for $S$. pombe $e^{59}$. To construct 3HA or GFP tagged Plo1 at its $\mathrm{N}$-terminus, the coding sequence of 3HA or GFP flanked by the 5'- and ORF containing 3'-untrasnrated region (UTR) of the plo1 gene was cloned into pUC119 (3HA; vp183 and GFP; pak64). The 5'-UTR-3HA/GFP-plo1-3'-UTR fragment amplified by PCR was transformed into temperature-sensitive plo1-ts $2:: u r a 4^{+}$strain (from Ian Hagan). To construct the plol-tev allele, the recognition sequence for TEV protease Glu-Asp-Leu-Tyr-Phe-Gln-Gly(-Ala-Ser) was inserted at both the 341 a.a. and 405 a.a. sites in pak64 or 5'-UTR-plo1-3'-UTR (untagged version) fragment cloned into pUC119 by site-directed mutagenesis. PCR-amplified fragment contacting the plol-tev allele was introduced into plo1-ts2::ura4 strain. The construction of Cnp3C-CFP-TEV was described previously ${ }^{23}$, while the promoter was exchanged from Padh 81 to meiosis-specific Prec 8 . The T101A mutation of moal was introduced into 5'-UTR-3Pk-moal-3'-UTR fragment cloned in pBluescript by using the KpnI and SacI sites by site directed mutagenesis. KpnI and SacI-digested fragment containing 5'-UTR-3Pk-moal-T101A-3'-UTR were introduced into moa1::ura $4^{+}$strain. To visualize tubulin, besides the pREP81-CFP-atb2 $2^{+}$, Padh13 (a weaker promoter version of the $\mathrm{P} a d h 1$ )-mCherry-atb2 ${ }^{+}$integrated at the $Z$ locus using the $n a t^{r}$ marker was used $^{60}$. To localize the Spo13 protein to kinetochores in fission yeast meiotic cells, the SPO13 ORF cloned from S. cerevisiae W303 genome was fused to the C-terminus of Cnp3C-CFP, and expressed by meiosis specific Pspo5del (authentic TATA sequence of Pspo5 was deleted to reduce the expression level, but it still shows a stronger expression level than that of Prec8). The resulting plasmid (pHBCS5del-Cnp3C-CFP-SPO13) was linearized and integrated at the $C$ locus ${ }^{60}$ using the $h y g^{r}$ marker. To construct spo13-5A, five STSTP residues (132 a.a. to 136 a.a.) of SPO13 gene in pHBCS5del-Cnp3C-CFP-SPO13 were substituted to alanines by site directed mutagenesis. To target Moal or the Moa1-T101A protein to 
kinetochores in fission yeast meiotic cells, $\mathrm{moal}^{+}$or moal-T101 A fragment were cloned into pHBCS5del-Cnp3C-CFP, and integrated at the $C$ locus using the $h y g^{r}$ marker.

\section{Synchronization of fission yeast meiotic cells}

For microscopic observation of cen2-GFP or GFP-Plo1, logarithmically growing cells were collected and suspended in $20 \mathrm{mg} / \mathrm{ml}$ leucine and spotted on SPA and incubated at $26.5^{\circ} \mathrm{C}$ for 12 15 h (cen2-GFP observation in spored asci) or 6 8 h (GFP-Plo1 observation at metaphase I zygote with short spindle). Fluorescence images were taken using a microscope (Axioplan2, Zeiss) equipped with a cooled CCD camera (Quantix, Photometrics) and AxioVision software (Zeiss). Seven Z sections (0.45 Mm each) for GFP signals were converted into single two-dimensional images by taking the maximum signal at each pixel position in the images. For immunoprecipitation analysis of Pk-Moa1, we used haploid cells containing the pat1-114 allele. Cells were grown in $\mathrm{MM}$ liquid medium including $\mathrm{NH}_{4} \mathrm{Cl}(\mathrm{MM}+\mathrm{N})$ to a density of $5 \times 10^{6}$ cells $/ \mathrm{ml}$ at $25^{\circ} \mathrm{C}$, then resuspended in $\mathrm{MM}$ medium lacking $\mathrm{NH}_{4} \mathrm{Cl}$ $(\mathrm{MM}-\mathrm{N})$ at a density of $1 \times 10^{7}$ cells $/ \mathrm{ml}$ at $25^{\circ} \mathrm{C}$ for $15 \mathrm{~h}$. To induce meiosis, cells were incubated at $34^{\circ} \mathrm{C}$, and collected cells $4 \mathrm{~h}$ after meiosis induction, the period of meiotic prophase $^{23}$.

\section{Immunoprecipitation from yeast extracts}

Before harvesting the meiotic cells, $1 \mathrm{mM}$ PMSF (phenylmethylsulfonyl fluoride, SIGMA) was added to reduce protein degradation. Cell extracts $\left(\sim 8 \times 10^{7}\right.$ cells $)$ were prepared by beating the cells with beads in HB IP buffer (25 mM MOPS, $5 \mathrm{mM} \mathrm{EGTA,} 15 \mathrm{mM} \mathrm{MgCl}$, $150 \mathrm{mM} \mathrm{KCl}, 50 \mathrm{mM}$ ß-glycerophosphate, $15 \mathrm{mM}$ p-nitrophenylphosphate, $1 \mathrm{mM}$ DTT, 0.1 $\mathrm{mM}$ sodium vanadate, $0.8 \% \mathrm{NP} 40,1 \mathrm{mM}$ PMSF). Immunoprecipitation was performed by incubating the whole cell extracts for $1 \mathrm{~h}$ at $4{ }^{\circ} \mathrm{C}$ with anti-Pk monoclonal antibody (Serotec), and $20 \mu \mathrm{l}$ of protein A-Sepharose (GE-healthcare) for $2 \mathrm{~h}$ at $4^{\circ} \mathrm{C}$. Whole cell extracts and immunoprecipitates were subjected to immunoblot analysis using mouse anti-HA monoclonal antibody 12CA5 (Roche) or anti-Pk monoclonal antibody.

51. Kim-Kaneyama, J.R. et al. Hic-5 deficiency enhances mechanosensitive apoptosis and modulates vascular remodeling. J Mol Cell Cardiol 50, 77-86 (2011).

52. Ishiguro, K., Kim, J., Fujiyama-Nakamura, S., Kato, S. \& Watanabe, Y. A new meiosis-specific cohesin complex implicated in the cohesin code for homologous pairing. EMBO Rep 12, 267-275 (2011). 
53. Kawashima, S.A., Yamagishi, Y., Honda, T., Ishiguro, K. \& Watanabe, Y. Phosphorylation of $\mathrm{H} 2 \mathrm{~A}$ by Bub1 prevents chromosomal instability through localizing shugoshin. Science 327, 172-177 (2010).

54. Morimoto, A. et al. A conserved KASH domain protein associates with telomeres, SUN1, and dynactin during mammalian meiosis. J Cell Biol 198, 165-172 (2012).

55. Chambon, J.P., Hached, K. \& Wassmann, K. Chromosome spreads with centromere staining in mouse oocytes. Methods Mol Biol 957, 203-212 (2013).

56. Hodges, C.A. \& Hunt, P.A. Simultaneous analysis of chromosomes and chromosome-associated proteins in mammalian oocytes and embryos. Chromosoma 111, 165-169 (2002).

57. Shibuya, H., Ishiguro, K. \& Watanabe, Y. The TRF1-binding protein TERB1 promotes chromosome movement and telomere rigidity in meiosis. Nat Cell Biol 16, 145-156 (2014).

58. Rabut, G. \& Ellenberg, J. Automatic real-time three-dimensional cell tracking by fluorescence microscopy. J Microsc 216, 131-137 (2004).

59. Bahler, J. et al. Heterologous modules for efficient and versatile PCR-based gene targeting in Schizosaccharomyces pombe. Yeast 14, 943-951 (1998).

60. Sakuno, T., Tanaka, K., Hauf, S. \& Watanabe, Y. Repositioning of Aurora B promoted by chiasmata ensures sister chromatid mono-orientation in meiosis I. Dev Cell 21, 534-545 (2011).

61. Nagaoka, S.I., Hodges, C.A., Albertini, D.F. \& Hunt, P.A. Oocyte-specific differences in cell-cycle control create an innate susceptibility to meiotic errors. Curr Biol 21, 651-657 (2011).

\section{Figure legends}

\section{Figure 1. Meiotic kinetochore protein MEIKIN.}

a, Schematic drawing of behavior of homologous chromosomes during meiosis; metaphase I (Meta I) and anaphase I (Ana I). b, Spermatocytes stained for MEIKIN, ACA, SYCP3 and DAPI (DNA); zygotene (Zygo), pachytene (Pachy), diplotene (Diplo), prometaphase I (Prometa I) and prometaphase II (Prometa II). c, Spermatocytes from the wild-type testis transfected with GFP-tagged MEIKIN stained for GFP, SYCP3 and DAPI (DNA). Alanine substitutions (5A and $4 \mathrm{~A}$ ) were introduced on the C-terminal conserved sequences of MEIKIN (also see Extended Data Fig. 2). d, WT and Meikin ${ }^{-/}$diplotene spermatocytes stained for MEIKIN and SYCP3. Enlarged images of a kinetochore. e, WT and Meikin ${ }^{-/-}$ round spermatids stained for CENP-C and DAPI (DNA). $* * * * P<0.0001$, unpaired $t$-test. 
Scale bars, $5 \mu \mathrm{m}$.

Figure 2. MEIKIN is required for the protection of centromeric cohesion during meiosis I.

a, Cumulative oocytes 1 st polar body extrusion rates after GV breakdown (GVBD). mean \pm s.e.m. from 4 independent experiments. Total oocytes 40 for wild-type, 58 for Meikin ${ }^{-/-}$. b, Metaphase I oocytes ( $6 \mathrm{~h}$ post GVBD) stained for $\alpha$-tubulin, CENP-C and DAPI (DNA) and the chromosome alignment widths were measured (mean + s.e.m. of three independent experiments). 10 oocytes in each experiment. c, Time-lapse imaging of the first meiotic division (h:mm) in oocytes expressing 2mEGFP-CENP-C and H2B-mCherry. The z-projection images at metaphase I and anaphase I (upper). The images were reconstructed in $3 \mathrm{D}$, and kinetochore tracks are indicated by grey lines (bottom). The sister kinetochore distances at anaphase I are color-coded as indicated. The ratios of separated sister kinetochores (distance $>1.2 \mu \mathrm{m}$ ) at anaphase I were measured in 3 oocytes (mean \pm s.d.). Also see video 1 in Supplementary Information. d, Time-lapse imaging of the second meiotic division (h:mm) in oocytes as in c (upper). Resumption of kinetochore movement after 1st PBE was assumed as the end of telophase I (Telo I). The number of kinetochores with distance $>3 \mu \mathrm{m}$ from the metaphase plate were counted over time (bottom). Also see video 2 in Supplementary Information. e, Oocytes at metaphase I and metaphase II stained for REC8, CENP-C and DAPI (DNA). The spread chromosomes at metaphase II were classified according to REC8 signals and kinetochore distance (mean \pm s.e.m. of three independent experiments). 5 oocytes in each experiment. f, Prometaphase I oocytes ( $4 \mathrm{~h}$ post GVBD) stained for SGO2, CENP-C and DAPI (DNA) in whole mount. The signal intensity of SGO2 adjacent to the centromere was quantified and normalized to that of CENP-C (mean + s.e.m. of three independent experiments). In each experiment, 15 centromeres from an oocyte were quantified ( $\mathrm{n}=5$ cells). ${ }^{*} P<0.05,{ }^{*} P<0.01, * * * * P<0.0001$, unpaired $t$-test $(\mathbf{b}, \mathbf{e}, \mathbf{f})$. Scale bars, $5 \mu \mathrm{m}$.

\section{Figure 3. MEIKIN regulates mono-orientation.}

a, WT and $\mathrm{Meikin}^{-/}$oocytes at early prometaphase I ( $2 \mathrm{~h}$ post GVBD) stained for CENP-C and DAPI (DNA). The pairs of sister kinetochores are magnified. Measurement of the distance between two CENP-C signals of a sister kinetochore pair on different z-planes (mean + s.e.m. of three independent experiments). In each experiment, 10 kinetochores were measured in a cell $(n=5$ cells). $\mathbf{b}$, Cumulative 1 st PBE rates after GVBD (mean \pm s.e.m. of three independent experiments). Total oocytes 45 for WT, 29 for $M l h 1^{-/-}$and 29 for $M l h 1^{-/-}$ Meikin $^{-/-}$. c, Oocytes at $10 \mathrm{~h}$ post GVBD stained for $\alpha$-tubulin, CENP-C and DAPI (DNA) in 
whole mount. Bi-oriented sister kinetochores; distance $>0.6 \mu \mathrm{m}$ with horizontal angle $\left(0-10^{\circ}\right)$. The number of bi-oriented sister kinetochores at the spindle midzone (10 $\mu \mathrm{m}$ width centered between spindle poles; only spindles up to $30 \mu \mathrm{m}$ in length were used) was scored in each cell. Note that the spindle length becomes longer in $M l h 1^{-/-}$because of less alignment of chromosomes $^{61}$. d, Metaphase I spermatocytes were stained and examined as in c. Spindle midzone, $6 \mu \mathrm{m}$ (only spindles up to $15 \mu \mathrm{m}$ in length were used). Sister kinetochores distance $>0.6 \mu \mathrm{m}$ as 'bi-oriented'. NS, not significantly different. $* * * P<0.001, * * * * P<0.0001$,

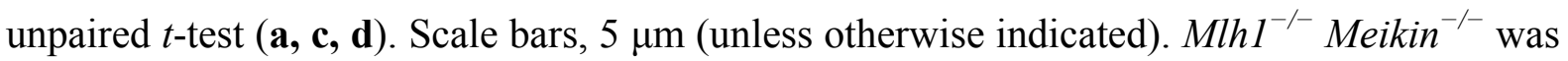
compared to littermates $M l h 1^{-/-}$with C57BL/6 background (c, d).

\section{Figure 4. PLK1 is required for mono-orientation and the protection of centromeric} cohesion.

a, Immunoprecipitates from mouse testis chromatin extracts. b, Spermatocytes stained for MEIKIN, PLK1, SYCP3 and DAPI (DNA). c, Prometaphase I oocytes ( $3 \mathrm{~h}$ post GVBD) stained for PLK1, ACA and DAPI (DNA). The relative intensities of PLK1 normalized to that of ACA (mean + s.e.m. of three independent experiments). In each experiment, 15 kinetochores from an oocyte were quantified $(n=4$ cells). d, A schematic time course of PLK1 inhibition experiment in wild-type oocytes (upper). The number of single chromatids at the spindle polar zone was scored at metaphase II (bottom). mean \pm s.e.m. of three independent experiments. Ten oocytes were counted in each experiment. e, The spread chromosomes at metaphase II were classified as in Fig. 2e. mean \pm s.e.m. of three independent experiments. 5 oocytes were counted in each experiment. $P$-values, unpaired $t$-test from REC8-negative categories. f, $M l h 1^{-/}$oocytes (C57BL/6 background) were treated with BI 2536 between 9-10 h post GVBD and bi-oriented sister kinetochores were scored as indicated in Fig. 3c. $* P<0.05, * * P<0.01, * * * P<0.001, * * * * P<0.0001$, unpaired $t$-test (c, d, e, f). Scale bars, $5 \mu \mathrm{m}$.

\section{Figure 5. S. pombe Moa1 and S. cerevisiae Spo13 are MEIKIN homologs.}

a, Schematic representation of mouse MEIKIN, S. pombe Moal and S. cerevisiae Spo13. Asterisks indicate the polo-box binding motif (STP). Shaded boxes indicate the CENP-C binding region. b, Co-precipitation of Moal and Plo1 in S. pombe meiotic cell extracts prepared from the indicated cells expressing Pk-Moa1 and HA-Plo1. c, Plo1-GFP localizes at kinetochores and SPB in metaphase I, while the kinetochore localization is abolished in moal-101A and moals cells. The spindles were visualized by expressing cyan fluorescent protein (CFP)-Atb2 ( $\alpha 2$-tubulin). Schematic representation of a metaphase I zygote is shown. d, The indicated zygotes examined for reductional (red.) or equational (eq.) segregation of 
heterozygous cen2-GFP during meiosis I. e, Schematic diagram of Cnp3C-TEV and Plo1-tev, in which two TEV protease-recognition sites were inserted between the kinase domain and polo-box domain (PBD). Representative images of GFP-Plo1-tev signals along the metaphase I spindle (mCherry-Atb2) in cells expressing Cnp3C-CFP or Cnp3C-CFP-TEV. f, Segregation of heterozygous cen2-GFP during meiosis I was examined in plo1-tev rec12 $\Delta$ zygotes with vector (-) or expressing Cnp3C-CFP-TEV (+). g, Segregation of heterozygous cen2-GFP during meiosis I was examined in the moals rec12 $\Delta$ zygotes expressing the indicated Cnp3C-fusion proteins. $\mathrm{n}>200$ zygotes each. $\mathbf{h}$, Schematic depiction of meiotic kinetochore regulation by meikin, which cooperates with PLK. Error bars, s.e.m. from 3 independent experiments; ${ }^{* *} P<0.05,{ }^{*} P<0.01$, unpaired $t$-test $(\mathbf{d}, \mathbf{f}, \mathbf{g})$. Fission yeast strains used in this work are listed in Supplementary Table 1.

\section{Extended Data Figure 1: MEIKIN (4930404A10rik) was identified as a meiosis-specific CENP-C binding protein.}

a, Yeast two-hybrid screening was performed using CENP-C C-terminus (a.a. 692-906) as bait and a mouse testis cDNA library as prey. $11.95 \times 10^{7}$ colonies were screened on selective (SD-Trp-Leu-His-Ade, $+10 \mathrm{mM} 3 \mathrm{AT}$ ) plates using the AH109 tester strain. The number of clones isolated by screening is summarized. $\mathbf{b}$, To search for a meiosis-specific candidate from the isolated clones, tissue specific expression patterns of CENP-C interactors were examined by RT-PCR. RNA was extracted from each tissue of both males and females. Testis RNA was derived from 8-week males. Ovary RNA was derived from 4- and 8-week females. RT(-) indicates control PCR without reverse transcription. Note that the expression of 4930404 A10rik is restricted to testis and ovary as that of $S M C 1 \beta$. c, Immunoprecipitates from mouse testis chromatin extracts using anti-CENP-C antibody or control IgG were analyzed by the indicated antibody. d, The C-terminal domain of MEIKIN (4930404A10rik) (a.a. 385-434) interacts with the CENP-C C-terminus in yeast two-hybrid assay.

\section{Extended Data Figure 2: Sequence alignment of MEIKIN homologs in vertebrates.}

Amino acid sequences of $M$. musculus 4930404A10Rik (NP_083381), R. norvegicus (XP_573090), C. lupus (XP_003639413), X. tropicalis (XP_002934413) and G. gallus (XP_001234011) are derived from the NCBI protein database. H. sapiens data is derived from cDNA clones. 4930404A10Rik protein is conserved among vertebrates but it does not have any known motif except for the polo-box binding motif (blue line) in mammalian proteins. Two-hybrid assays indicate that this motif of mouse MEIKIN is important for PLK1 binding (data not shown), although the motif is apparently not conserved in Xenopus and chicken. The C-terminal sequences (red lines) are required for the kinetochore localization 
(see Fig. 1c).

\section{Extended Data Figure 3: Colocalization of CENP-C and ACA in spermatocytes and MEKIN localization in oocytes.}

a, Squashed spermatocytes from wild-type were immunostained for CENP-C, ACA, SYCP3 and DAPI at the indicated stages during meiosis. CENP-C and ACA signals accumulate and colocalize at centromeres after zygotene throughout meiosis. b, Chromosome spreads of oocytes from wild-type were immunostained for MEIKIN, ACA and REC8 at different meiotic stages. Zygotene from E15.5, Pachytene and Diplotene from E18.5 mouse. c, Chromosome spreads of oocytes at metaphase I ( $5 \mathrm{~h}$ post GVBD) and metaphase II (16 h post GVBD) were stained for MEIKIN, ACA and DAPI. Scale bars, $5 \mu \mathrm{m}$.

\section{Extended Data Figure 4: Generation of Meikin-knockout mice.}

a, Schematic illustrations of the wild-type allele and targeted $\mathrm{Meikin}^{-/-}$allele are shown. Grey boxes represent exons. The targeted exon 4 contains the intact splicing acceptor (SA) sequence followed by a premature stop codon, resulting in disruption of the Meikin allele. Black bar probe for Southern blot. b, Southern blot of genomic DNA from wild type $(+/+)$ and Meikin heterozygous (+/-) ES cells after Pvu II digestion. ES cell clone \#2 used to generate the mice. c, Immunoblot analysis of testis extracts prepared from mice with the indicated genotypes (4-week-old). In $\mathrm{Meikin}^{-/-}$, the specific bands probed by the anti-MEIKIN antibody are absent (shown as arrowheads). $\alpha$-tubulin is a loading control. d, Testes from 12 week-old wild-type (+/+) and $\mathrm{Meikin}^{-/-}(-/-)$mice. e, Hematoxylin-eosin staining of a section of the testis (12-week-old) showed seminiferous tubules. Enlarged pictures of seminiferous tubules showed spermatocyte (black arrowheads) and spermatids (yellow arrowheads) in wild-type and $\mathrm{Meikin}^{-/}$. Scale bar, $100 \mu \mathrm{m}$. f, Hematoxylin-eosin staining of epididymis from 12-week-old mice shows reduced number of sperms in Meikin ${ }^{-/}$. Enlarged images of sperms are shown. Scale bar, $50 \mu \mathrm{m}$. g, A pair of ovaries (8-week-old) from the indicated genotypes (top). Hematoxylin and eosin-stained paraffin sections of ovaries from 8-week-old wild-type and $\mathrm{Meikin}^{-/-}$mice (middle). The antral-stage follicles with oocyte nuclei are magnified (bottom). Asterisks indicate corpora lutea. Scale bar, 500 $\mu \mathrm{m}$.

\section{Extended Data Figure 5: The delay of anaphase I onset in $\mathrm{Meikin}^{-/-}$oocytes is canceled by inactivation of SAC.}

Oocytes from wild-type and Meikin ${ }^{-/-}$mice were cultured after GVBD in the presence of nocodazole $(10 \mu \mathrm{M})$ or the Mps1 kinase inhibitor reversin $(5 \mu \mathrm{M})$. The first polar body 
extrusion (PBE) rates are shown with mean \pm s.e.m. from 3 independent experiments. Total number of oocytes is 27 in WT with nocodazole (7, 10 and 10, respectively), 27 for WT with reversine (6, 10 and 11. respectively), 21 for Meikin $^{-/}$with nocodazole $(5,8$ and 8, respectively) and 23 for Meikin ${ }^{-/-}$with reversine (7, 8 and 8, respectively).

\section{Extended Data Figure 6: MEIKIN is required for centromeric cohesion protection and mono-orientation in spermatocytes.}

a, Squashed spermatocytes at metaphase I (Meta I) were immunostained for SGO2, ACA and DAPI. Partial z-projection images of aligned chromosomes are shown with magnified images of a bivalent (upper). The signal intensity of SGO2 adjacent to the centromere was quantified and normalized to that of ACA. The relative intensities are shown with mean + s.e.m. from 3 independent experiments (bottom). In each experiment, 15 centromeres from a spermatocyte were quantified $(n=5$ cells). b, Squashed spermatocytes at anaphase I ( $5 \mu \mathrm{m}<$ segregated DNA mass distance $<10 \mu \mathrm{m}$ ) from wild-type, $\mathrm{Meikin}^{-/-}$and $\mathrm{Sgo}^{-/-}$mice were immunostained for CENP-C, REC8 and DNA (upper). A pair of sister kinetochores is magnified. The distance between sister kinetochores was scored and represented in the scatter plot with median (bottom). 15 kinetochores from 5 spermatocytes were measured in each group ( $\mathrm{n}=$ kinetochore number). c, Squashed spermatocytes at prometaphase I from wild-type and $\mathrm{Meikin}^{-/-}$mice, and MEF cells at prometaphase (prometa) were immunostained for CENP-C and DNA. Pairs of sister kinetochores are magnified. The distances between sister kinetochores were scored and represented in the graph with mean + s.e.m. from three independent experiments (right). Note that, MEF cells sample preparation and sister kinetochore distance measurements were performed same methods with spermatocytes. In each experiment, 10 kinetochores were measured in a cell ( $\mathrm{n}=5$ cells). ${ }^{*} P$ $<0.05, * * * P<0.001, * * * * P<0.0001$, unpaired $t$-test (a, b, c). Scale bars, $5 \mu \mathrm{m}$ (unless otherwise indicated).

\section{Extended Data Figure 7: PLK1 was identified as a MEIKIN interactor.}

a, Yeast two hybrid screening of mouse MEIKIN interactors was performed using a mouse testis cDNA library. The number of clones isolated by screening is summarized. Because the use of MEIKIN full length and N-terminal region (a.a. 1-271) as bait resulted in a high background of false positive interactions in yeast two-hybrid screening, we used MEIKIN C-terminus (a.a. 272-434) as bait. b, Yeast two hybrid assay demonstrates that MEIKIN interacts directly with mouse PLK1 through the MEIKIN C-terminal domain (a.a. 272-434). c, Mouse MEIKIN protein was immunoprecipitated from testis chromatin-bound fraction by two different anti-MEIKIN polyclonal antibodies (A and B). The immunoprecipitates 
underwent two independent LC-MS/MS analyses. Those proteins commonly identified in all the LC-MS/MS analyses are listed with the number of peptide hits in the table.

Note that polo-like kinase (PLK1) as well as CENP-C were repeatedly identified in the two-hybrid screening (a) and LC-MS/MS analyses (c).

\section{Extended Data Figure 8: BI2536 treatment reduces PLK1 kinase activity in oocytes.}

a, Schematic illustration of BI2536 treatment in wild-type oocytes culture (left). Oocytes were treated with DMSO or BI $2536(100 \mathrm{nM})$ during the indicated time periods, then washed and released into normal culture medium. 1st polar body extrusion ratio (1st PBE) was counted at $10 \mathrm{~h}$ after GVBD (right). Error bars, mean \pm s.e.m. from 3 independent experiments. The total number of oocytes used for each experiment is shown. b, Wild-type oocytes treated with DMSO or BI2536 (during 6-7 $\mathrm{h}$ post GVBD) were fixed and immunostained for PLK1 substrate pCENP-U, ACA and DAPI at metaphase I (upper). The relative pCENP-U intensity normalized to that of ACA is shown in the graph with mean + s.e.m. from 3 independent experiments. In each experiment, 10 kinetochores from an oocyte were quantified ( $\mathrm{n}=4$ cells). ${ }^{* *} p<0.01$, unpaired $t$-test. c, Oocytes were treated with DMSO or BI 2536 during the period of 4-6 h post GVBD and stained for $\alpha$-tubulin, CENP-C and DAPI (DNA) at the indicated stages in whole mount (related to Fig. 4d). Magnified images are shown to highlight the separation of sister kinetochores in BI 2536-treated oocyte in anaphase I. d, Chromosome spreads from control and BI 2536-treated wild-type oocytes at metaphase I ( $6 \mathrm{~h}$ post GVBD) and metaphase II (20 h post GVBD) were stained for REC8, CENP-C and DAPI (DNA) (related to Fig. 4d). Magnified images are shown to highlight the loss of cohesion in BI 2536-treated oocytes in metaphase II. e, Mlh1 ${ }^{-/}$oocytes (C57BL/6 background) were treated with BI 2536 between $9-10 \mathrm{~h}$ post GVBD and stained for $\alpha$-tubulin, CENP-C and DAPI (DNA) in whole mount (related to Fig. 4e). Magnified images are shown to highlight bi-oriented sister kinetochores at the spindle midzone. Scale bars, $5 \mu \mathrm{m}$ (unless otherwise indicated).

\section{Extended Data Figure 9: The human homologue of MEIKIN.}

a, Schematic illustrations of mouse and human MEIKNs. The putative amino acid sequence of hMEIKIN full length (373 a.a.) was deduced from our own sequencing of DNA(see Extended Data Figure 2), which was amplified by RT-PCR from the human testis cDNA. The amino acid sequence equivalent to mouse Ex10 (mEX10) is absent in the hMEIKIN protein, despite our attempts at a computational search to identify the missing DNA sequence. b, Yeast two-hybrid assays demonstrate that the hMEIKIN C-terminus interacts with human 
CENP-C (full length), hCENP-C C-terminal (CENPC motif + Mif2 motif, a.a. 732-945) and $\mathrm{hPLK} 1$, in agreement with mouse MEIKIN data. c, Immunostaining of a human seminiferous tubule section (purchased from Biochain) demonstrates that hMEIKIN localizes to centromeres (ACA) in pachytene spermatocytes. We used anti-hMEIKIN-N and anti-hMEIKIN-C antibodies. Enlarged images of the rectangles are shown to highlight the co-localization of ACA and hMEIKIN (bottom). Scale bar, $5 \mu \mathrm{m}$. 

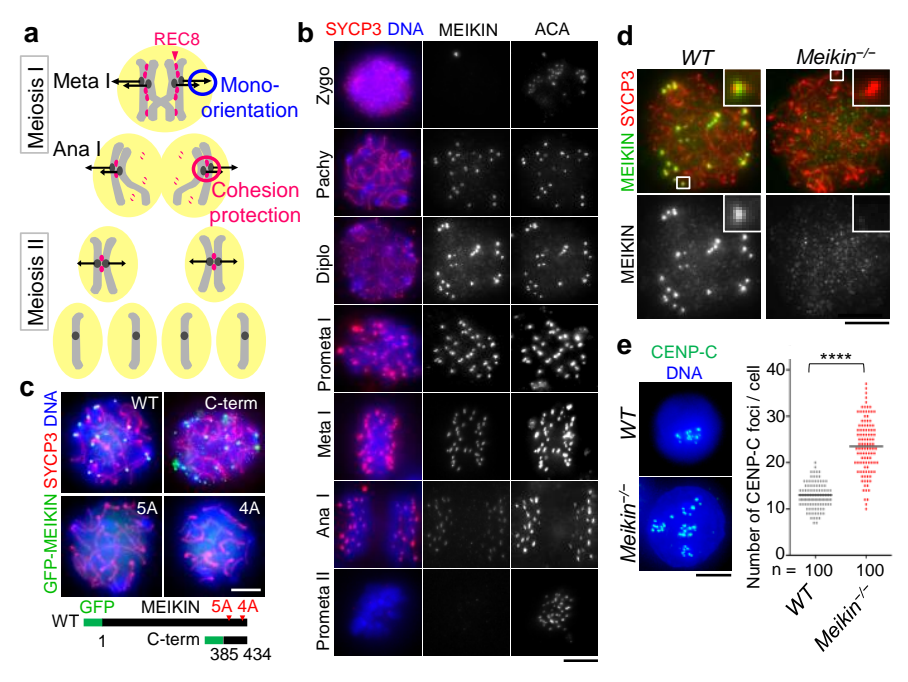

Figure 1 


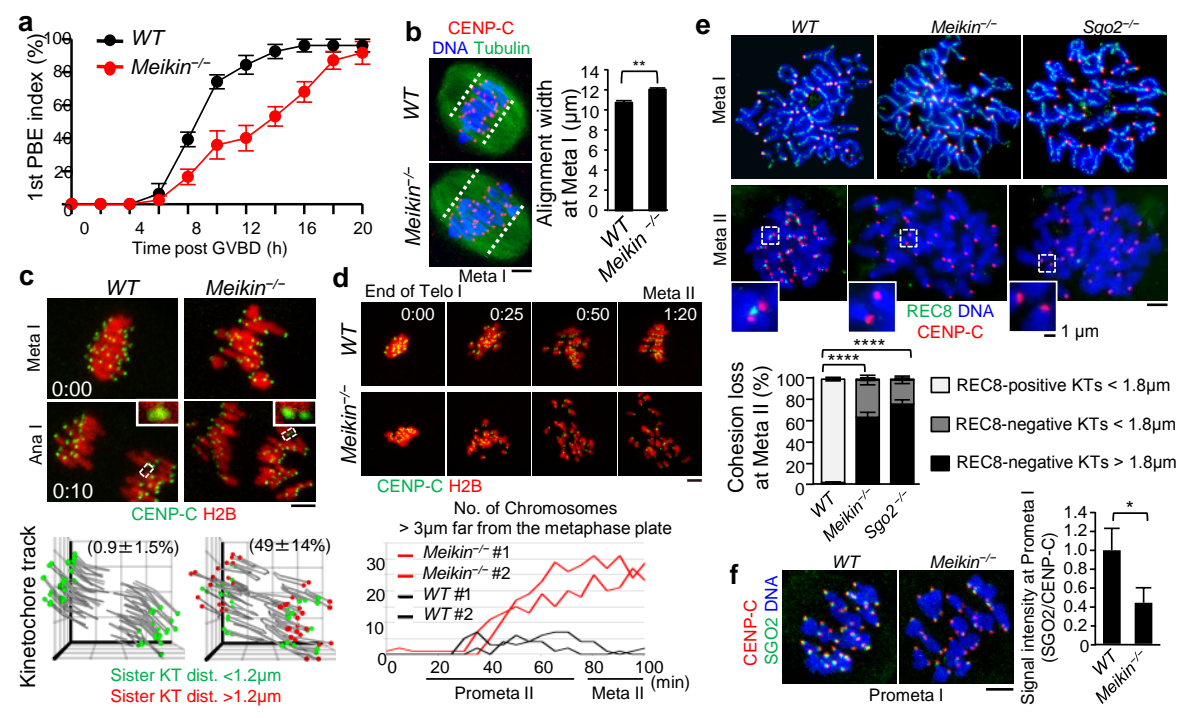


Figure 3

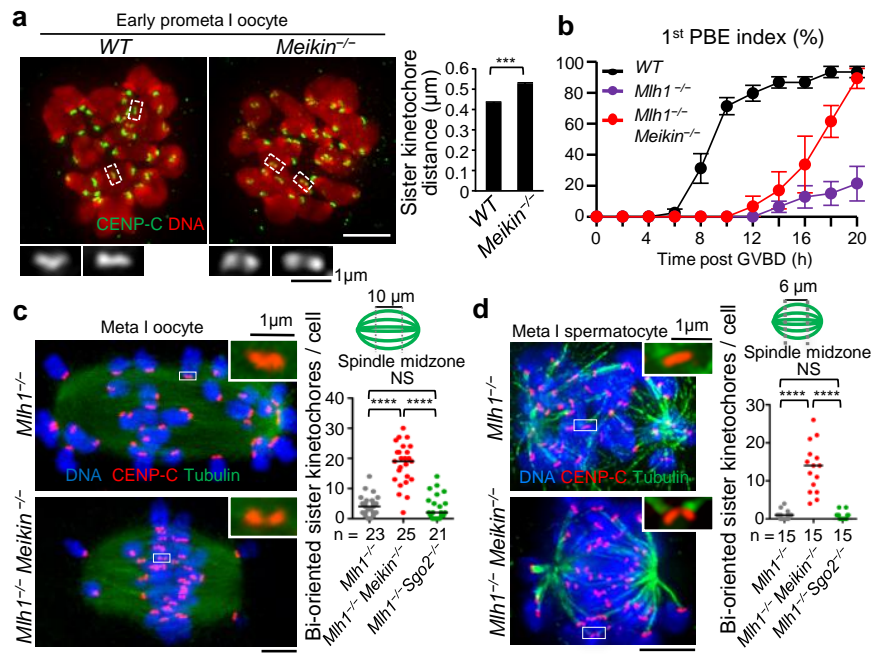


Figure 4

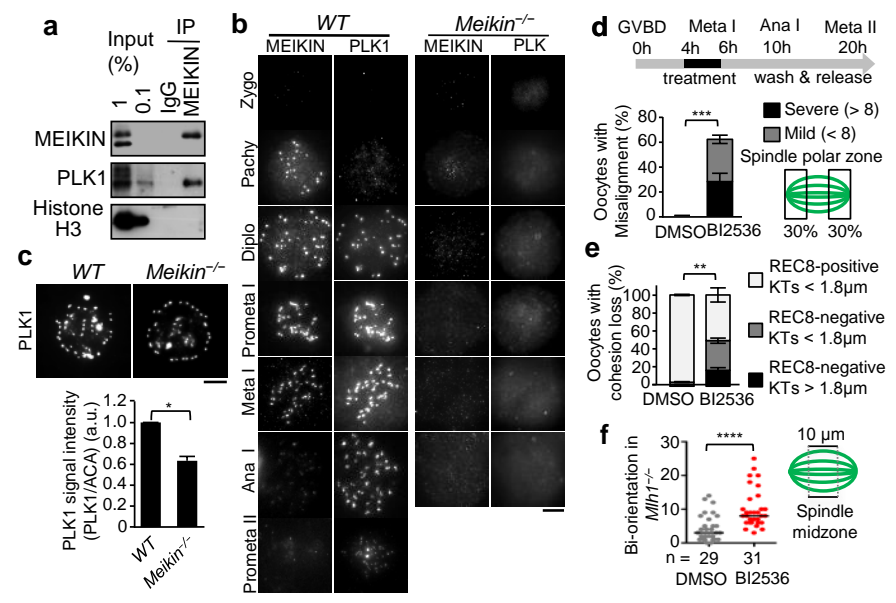


Figure 5

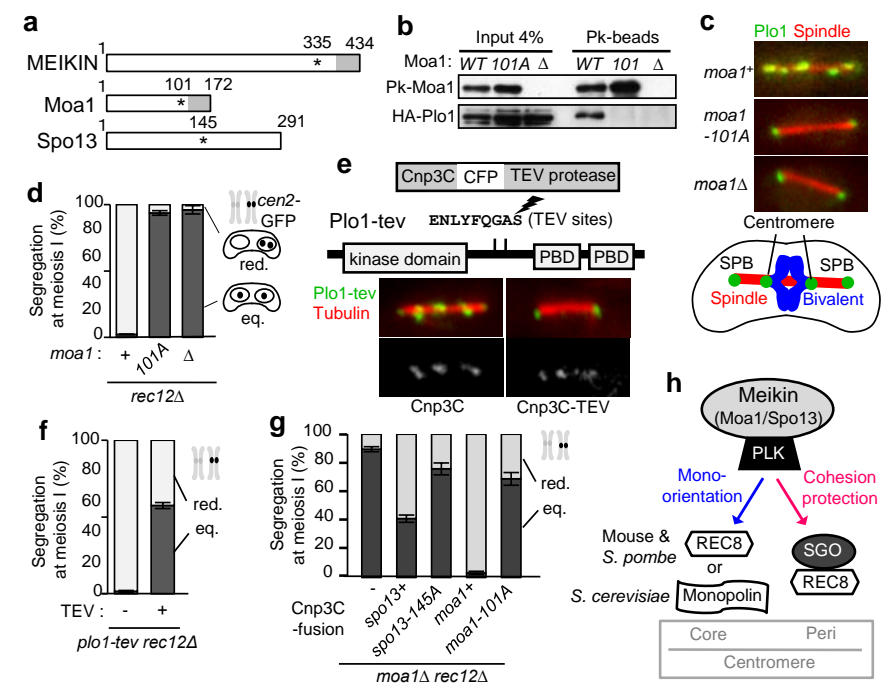

